\title{
Situação epidemiológica da tuberculose na cidade de Santos-SP no período de 2006 -
}

\section{6}

\author{
Epidemiological situation of tuberculosis in the city of Santos - SP during 2006-2016 \\ Situación epidemiológica de tuberculosis em la ciudad de Santos - SP durante 2006-2016
}

Recebido: 17/12/2021 | Revisado: 24/12/2021 | Aceito: 27/12/2021 | Publicado: 05/01/2022

\author{
Matheus Budahazi Jardine \\ ORCID: https://orcid.org/0000-0001-6041-406X \\ Centro Universitário Lusíada, Brasil \\ E-mail: matheusbudahazi@gmail.com \\ João Guilherme Saenz Carneiro \\ ORCID: https://orcid.org/0000-0003-2397-7802 \\ Centro Universitário Lusíada, Brasil \\ E-mail: jgsc13@gmail.com \\ Lucca Moreira Lopes \\ ORCID: https://orcid.org/0000-0002-2770-2198 \\ Centro Universitário Lusíada, Brasil \\ E-mail: lopeslucca015@hotmail.com \\ Hugo Garcia Fortunato \\ ORCID: https://orcid.org/0000-0002-0179-1516 \\ Centro Universitário Lusíada, Brasil \\ E-mail: hgfortunato@gmail.com \\ Marcos Montani Caseiro \\ ORCID: https://orcid.org/0000-0003-2950-4299 \\ Centro Universitário Lusíada, Brasil \\ E-mail: mcaseiro@uol.com
}

\begin{abstract}
Resumo
Análise estatística e da situação epidemiológica observada no município de Santos - SP entre 2006 e 2016. Avaliar a distribuição espacial dos casos de tuberculose ao longo dos anos e correlacionar a Tuberculose com demais variáveis epidemiológicas. Estudo de delineamento ecológico e tendencia temporal que visa avaliar estatisticamente os 4533 casos de Tuberculose. A análise dos resultados foi centralizada nas variáveis: Sexo, Encerramento, Drogadição e Municípios. Houve uma redução de 3\% no número de casos entre 2006-2016. Com relação ao desfecho dos pacientes diagnosticados com Tuberculose, $72,7 \%$ dos casos foram curados na década avaliada, $4,75 \%$ faleceram devido a Tuberculose e 3,2\% faleceram de demais causas. A taxa de abandono de tratamento foi de $15,2 \%$. Os bairros que apresentaram maior número de casos ao longo dos dez anos foram: Rádio Club (354), Vila Matias (257), Vila Nova (220). O município de Santos apresentou uma variação no número de casos anuais ao longo do período estudado, com momentos de aumento e de redução no número de casos, mas com estabilização nos últimos anos avaliados.
\end{abstract}

Palavras-chave: Tuberculose; Epidemiologia; Prevalência.

\begin{abstract}
Statistical analysis and the epidemiological situation observed in the city of Santos - SP between 2006 and 2016. To assess the spatial distribution of tuberculosis cases over the years and to correlate Tuberculosis with other epidemiological variables. Study of ecological design and temporal trend that aims to statistically evaluate 4533 cases of Tuberculosis. The analysis of the results was centered on the variables: Gender, Closure, Drug addiction and Municipalities. There was a 3\% reduction in the number of cases between 2006-2016. Regarding the outcome of patients diagnosed with Tuberculosis, $72.7 \%$ of the cases were cured in the decade evaluated, $4.75 \%$ died due to Tuberculosis and $3.2 \%$ died from other causes. The treatment dropout rate was $15.2 \%$. The neighborhoods with the highest number of cases over the ten years were: Radio Club (354), Vila Matias (257), Vila Nova (220). The municipality of Santos presented a variation in the number of annual cases over the period studied, with times of increase and decrease in the number of cases, but with stabilization in the last years evaluated.
\end{abstract}

Keywords: Tuberculosis; Epidemiology; Prevalence.

\section{Resumen}

Analizar estatística y situación epidemiológica observada en el municipio de Santos - SP entre 2006 y 2016. Avaliar a distribuição espacial dos casos de tuberculose ao longo dos anos e correlacionar a Tuberculose com demais variáveis epidemiológicas. Estudo de delineamento ecológico y tendencia temporal que visa avaliar estatisticamente os 4533 
casos de Tuberculose. A análise dos resultados foi centralizada nas variáveis: Sexo, Encerramento, Drogadição e Municípios. Houve uma redução de 3\% no número de casos entre 2006-2016. Com relação ao desfecho dos pacientes diagnosticados com Tuberculose, $72,7 \%$ dos casos foram curados na década avaliada, 4,75\% faleceram devido a Tuberculose y 3,2\% faleceram demais causas. Una taxa de abandono de tratamento foi de 15,2\%. Os bairros que apresentaram maior número de casos ao longo dos dez anos foram: Rádio Club (354), Vila Matias (257), Vila Nova (220). O município de Santos apresentou uma variação no número de casos anuais ao longo do período estudado, con momentos de aumento y reducción de número de casos, más con estabilización de los últimos años avaliados.

Palabras clave: Tuberculosis; Epidemiologia; Prevalência.

\section{Introdução}

Segundo o Global Tuberculosis Report 2017 da Organização Mundial de Saúde (OMS), a tuberculose é a nona maior causa de morte no mundo. No ano de 2016, 10,4 milhões de pessoas manifestaram a doença e 1,7 milhão vieram a óbito devido a esta. Estima-se que $25 \%$ da população mundial apresente a forma latente da TB e que 5 a $15 \%$ desta irá desenvolver manifestações clínicas ao longo da vida. A OMS aponta uma incidência de $2 \%$ ao ano e uma taxa de mortalidade de $3 \%$. Entretanto o risco de indivíduos imunodeprimidos, como aqueles que vivem com o HIV, diabéticos, fumantes e subnutridos de desenvolver a doença e até mesmo provocar a forma grave da doença é muito maior (OMS, 2017).

Caracterizando a Tuberculose no Brasil, o boletim epidemiológico de 2019 disponibilizado pela Secretária de Vigilância em Saúde (Ministério da Saúde, 2019), traçou o panorama nacional da doença. Segundo esse boletim houve 76 mil novos casos de Tuberculose diagnosticados e 4,5 mil mortes. De acordo com a nova classificação da OMS o Brasil ocupa a vigésima posição na lista dos 30 países prioritários para TB. O coeficiente de incidência acumulado entre 2001 e 2018 mostrou que houve uma redução na incidência, passando de 42,2 casos para cada 100 mil habitantes para 36,6 casos (Arakaki et al., 2011). Essa redução nacional observada não condiz com a realidade do município de Santos para os 10 anos avaliado (2006-2016), onde houve um aumento de 3\% na incidência local da doença.

Uma das grandes questões que contribuem para menores taxas de sucesso de tratamento está relacionada a taxa de abandono de tratamento (Mendes, 2004). Configura-se abandono de tratamento quando o paciente permanece faltoso por mais de 60 dias após a data da última consulta ou quando deixa de tomar o medicamento por 30 dias ou mais (). Segundo a Biblioteca Virtual em Saúde (BVS), o paciente que retorna ao serviço após reportado abandono de tratamento deve ter sua doença confirmada através de uma nova coleta de Baciloscopia. Além disso, se reinicia imediatamente o esquema inicial de tratamento deste paciente após coleta de novos exames ( Chirinos \& Meirelles, 2011).

$\mathrm{Na}$ tentativa de entender as razoes que levam os pacientes a abandonar o tratamento da Tuberculose, MENDES et al avaliaram um grupo de 340 pacientes com diagnostico confirmado de Tuberculose. Esse trabalho mostrou que o esquema terapêutico RHZ (Rifampcina, Isoniazida e Pirazinamida) foi utilizado por $88 \%$ dos pacientes que abandonaram o tratamento, quando houve uso do esquema RHZ, 32\% dos pacientes interromperam o tratamento com 1-3 meses e 24\% abandonaram com 3-5 meses de tratamento. A média geral observada foi de 3,69 meses de tratamento realizado. Este estudo ainda forneceu um questionário aos pacientes onde as principais causas apontadas para o abandono foram relacionadas ao tempo de tratamento, estado de saúde, influência familiar e agravos associados (Mendes et al., 2004).

O presente estudo possui como principais objetivos: Analisar a variação na incidência de Tuberculose no município de Santos - SP no período compreendido de 2006-2016. Avaliar a distribuição espacial dos casos de tuberculose ao longo dos anos e correlacionar a Tuberculose com demais variáveis epidemiológicas. 


\section{Metodologia}

O trabalho apresentado tem como tema: "A situação epidemiológica da Tuberculose na cidade de Santos - SP de 2006-2016" e foi desenvolvido durante o ano de 2021, pelos membros do núcleo de pesquisa de doenças de transmissão urbana, orientado pelo Prof. Dr. Marcos Montani Caseiro, do curso de Medicina do Centro Universitário Lusíada.

\section{Tipo de pesquisa}

Trata-se de um estudo de delineamento ecológico e tendencia temporal, pois combina estudo de abordagem das relações entre a saúde e o espaço ao longo de um período através do emprego de variáveis qualitativas (Pereira A. S. et al. 2018). Visa descrever os casos de Tuberculose no município de Santos e, em um segundo momento comparando os dados obtidos com a Baixada Santista e com o estado de São Paulo, relacionando os dados epidemiológicos observados com as variáveis selecionadas.

\section{Local da pesquisa}

A pesquisa foi realizada no Centro Universitário lusíada com auxílio e disponibilização dos dados pelo Centro de vigilância epidemiológica do estado de São Paulo (CVE-SP) no periodo de 2006-2016. Os dados de cada município da Baixada Santista foram analisados e pautados nos coeficientes de incidencia para cada ano.

\section{Amostra}

A partir da base de dados cedida pelo CVE, foi realizada uma divisão do número de casos/ano para cada município da Baixada Santista (Santos, Guarujá, Peruíbe, Itanhaém, São Vicente, Cubatão, Mongaguá, Bertioga e Praia Grande) seguida de uma seleção apenas dos dados referentes a cidade de Santos. Após essa seleção obteve-se uma amostra de 4533 pacientes para o período de 2006-2016.

\section{Análise e Métodos estatísticos}

Amostra total obtida de 4533 pacientes, todos diagnosticados com Tuberculose entre 2006-2016.

Variáveis epidemiológicas analisadas:

a. Sexo: Definido como masculino (M) ou feminino (F).

b. Encerramento: São 7 encerramentos possíveis, sendo eles: Cura, abandono, falência/resistência, mudança de diagnóstico, óbito TB, óbito NTB e Transf outro estado/país.

c. Municípios: Santos, Guarujá, Peruíbe, Itanhaém, São Vicente, Cubatão, Mongaguá, Bertioga e Praia Grande.

d. Drogadição: Definida por Sim ou Não.

e. HIV: Definida por Não realizado, sem informação, Positivo e Negativo.

Para confecção das análises estatísticas e dos gráficos, foi utilizada a plataforma Microsoft Excel.

Aspectos Éticos: o trabalho foi submetido e aprovado pelo Comitê de Ética em Pesquisa com seres humanos do Centro Universitário Lusíada (Santos) em 24 de setembro de 2018 - São Paulo. A declaração de Anuência foi obtida junto a Divisão de Tuberculose, com sede a Av. Arnaldo, 351 - São Paulo no Centro de Vigilância Epidemiológica (CVE-SP). 


\section{Resultados}

Tendo em vista uma análise não apenas da cidade de Santos, mas de todo o território que compõem a baixada, em um primeiro momento consideramos os resultados obtidos para todos os 9 municípios que compõem a Baixada Santista, a fim de realizar uma comparação de toda essa região com a cidade de Santos - SP.

Figura 1 - Casos de Tuberculose na cidade de Santos entre 2006-2016

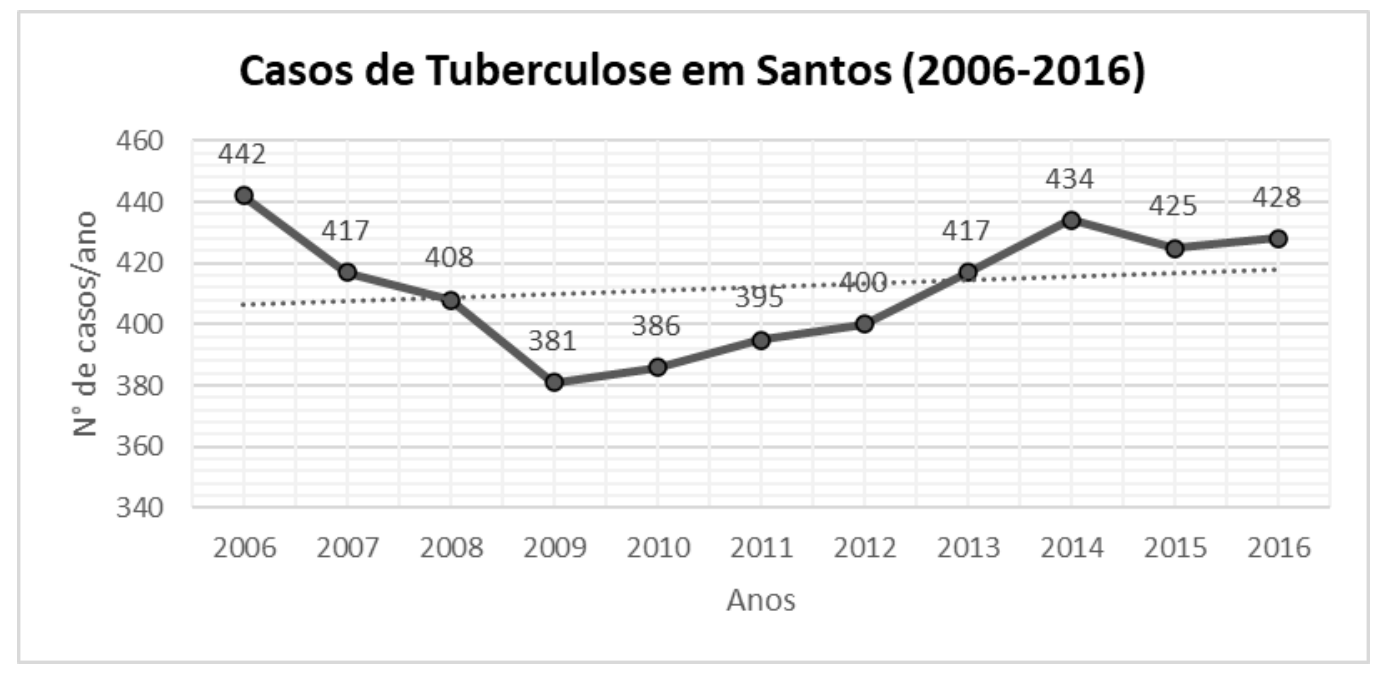

Fonte: Autores.

Avaliando especificamente a cidade de Santos - SP, observamos que houve uma queda no número anual de casos da doença no início nesta década estudada na Figura 1. Podemos dividir esta década em 3 momentos diferentes, fase de queda significativa no número de casos (2006-2009), fase de aumento significativo (2009-2014) e fase de leve queda (2014-2016). No ano de 2006, Santos apresentou 442 novos casos de Tuberculose, havendo uma queda significativa até o ano de 2009 com 381 casos novos no município. Após 2009, houve um aumento progressivo no número de caos até o ano de 2014, passando para 434 casos. Por fim, houve uma leve queda no terceiro período estudado, partindo para 428 casos em 2018.

Em relação a toda década estudada, houve uma redução de 3\% entre 2006-2016. Observando apenas entre 2006 e 2009, houve uma queda de 13\% no número de casos, entre 2009 e 2014 houve um aumento de 13,9\%. Por fim, entre 2014 e 2016 tivemos uma nova queda registrada de $1,3 \%$.

Um importante dado acerca da Tuberculose está relacionado ao tipo de encerramento obtido, ou seja, qual foi o desfecho para os pacientes que receberam o diagnóstico de TB durante o período avaliado. A variável "Encerramento" foi considerada com os seguintes desfechos: Cura, mudança de diagnóstico, abandono, falência/resistência, óbito TB, óbito NTB, transf, outro estado/país. 
Figura 2 - Tipo de Encerramento dos casos diagnosticados com Tuberculose entre 2006-2016.

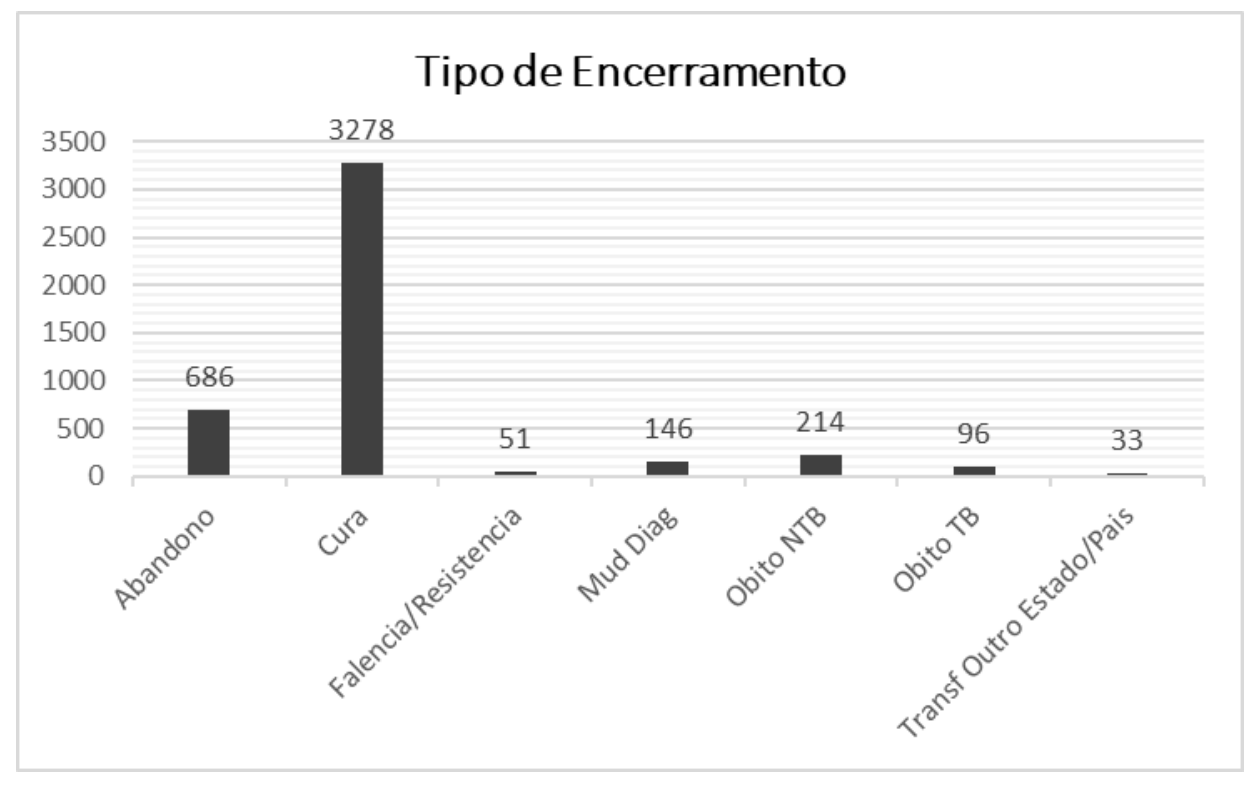

Fonte: Autores.

Analisando os 7 desfechos possíveis para "Encerramento" na Figura 2, notamos que 72,7\% dos casos foram curados na década avaliada, 4,75\% faleceram devido a Tuberculose e 3,2\% faleceram de demais causas. A taxa de falência de tratamento representou $1,1 \%$ da população total, mostrando a efetividade do tratamento adequado. Contudo, um dado muito preocupante observado está relacionado a taxa de abandono de tratamento, 15,2\% dos pacientes diagnosticados com Tuberculose iniciaram o tratamento e não o finalizaram, representando o grupo abandono de tratamento.

Figura 3 - Taxa de cura após tratamento da Tuberculose entre 2006-1016.

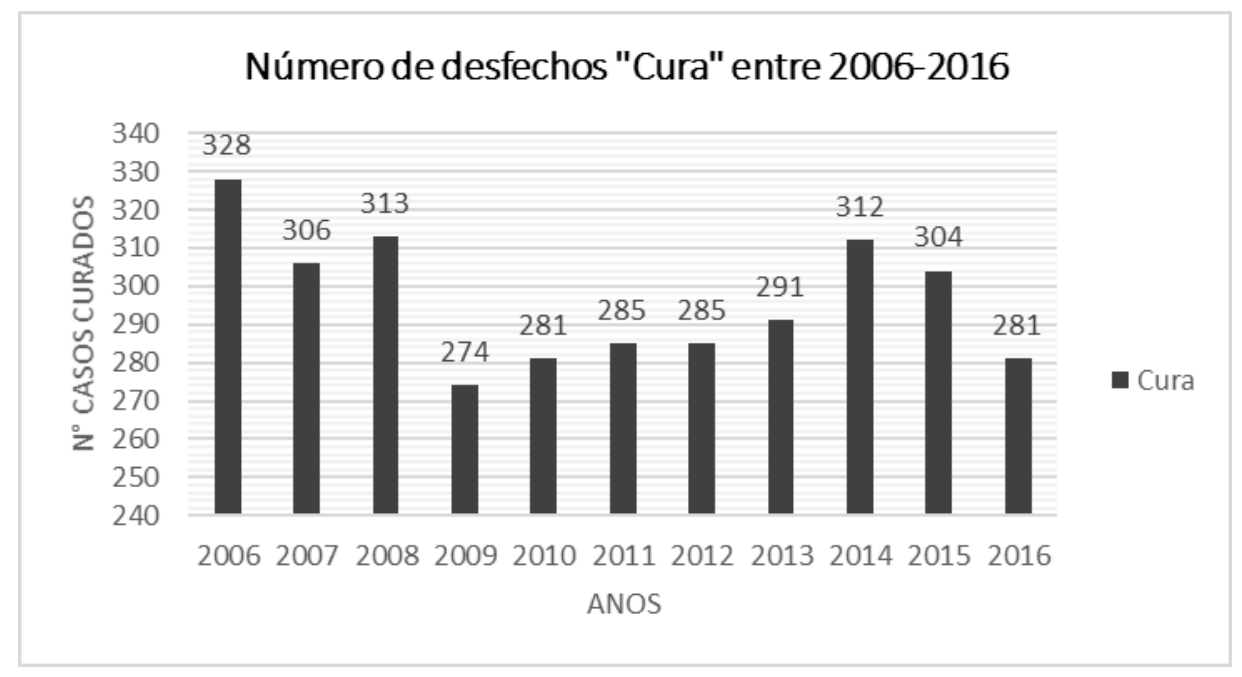

Fonte: Autores.

Iniciando a análise de cada uma das variáveis obtidas para o desfecho "Encerramento", verificamos que a taxa de cura apresentou grande variação ao longo da década estudada. Através da análise da Figura 3 vemos que houve uma queda no número de pacientes curados após tratamento, partindo de 328 pacientes curados em 2006 para 281 em 2016 , representando uma queda de $14 \%$ na década. A maior redução observada ocorreu entre o ano de 2008 e 2009, com uma queda de $13 \%$. 
Figura 4 - Taxa de abandono de tratamento da Tuberculose entre 2006-2016.

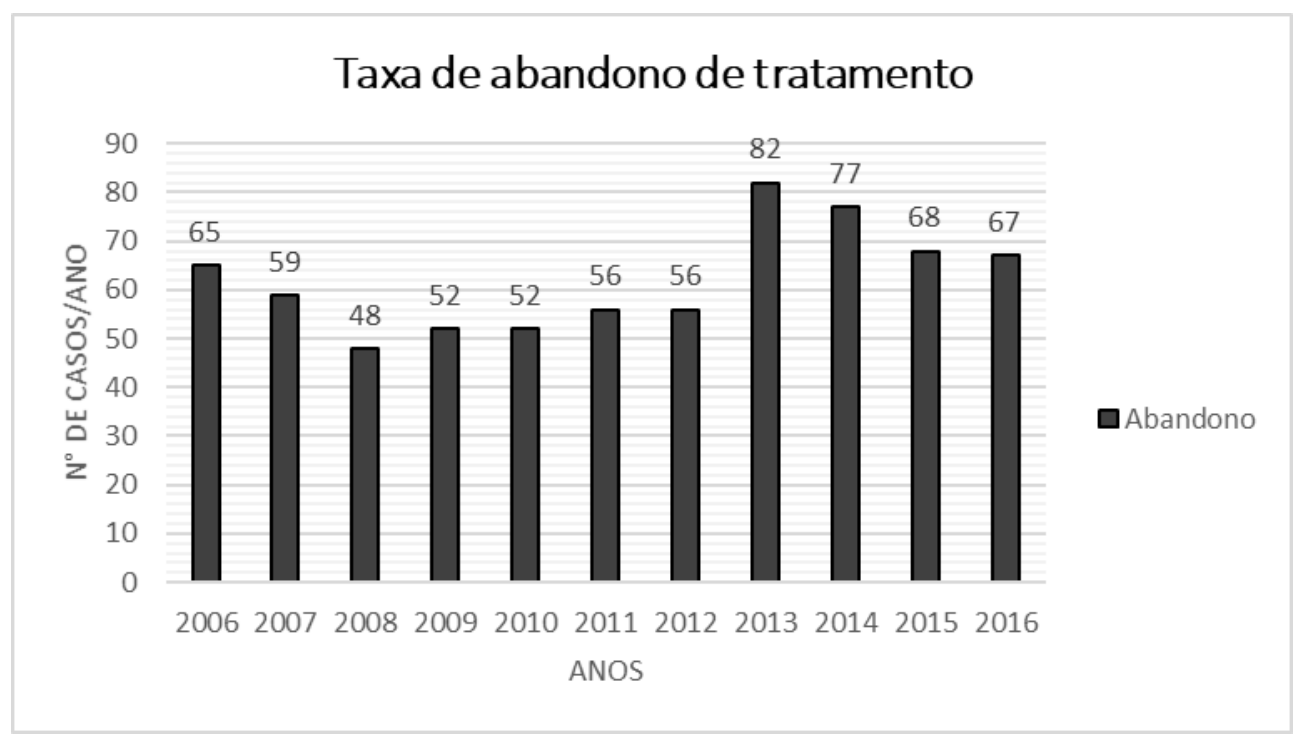

Fonte: Autores.

Uma variável que prediz sucesso em manter o paciente em tratamento é a taxa de abandono. Ao avaliarmos essa taxa para o município de Santos através da Figura 4, notamos que houve um leve aumento no abandono de tratamento para o período total estudado. Em um primeiro momento observamos uma queda no número de casos, partindo de 65 em 2006 para 48 em 2008 (maior queda observada), a partir disso observamos uma elevação do número de casos de 2008 até 2013, saltando de 48 casos para 82. A maior taxa de abando observada ocorreu em 2013 (82 casos).

Figura 5 - Taxa de abandono de tratamento dividido por sexo entre 2006-2016.

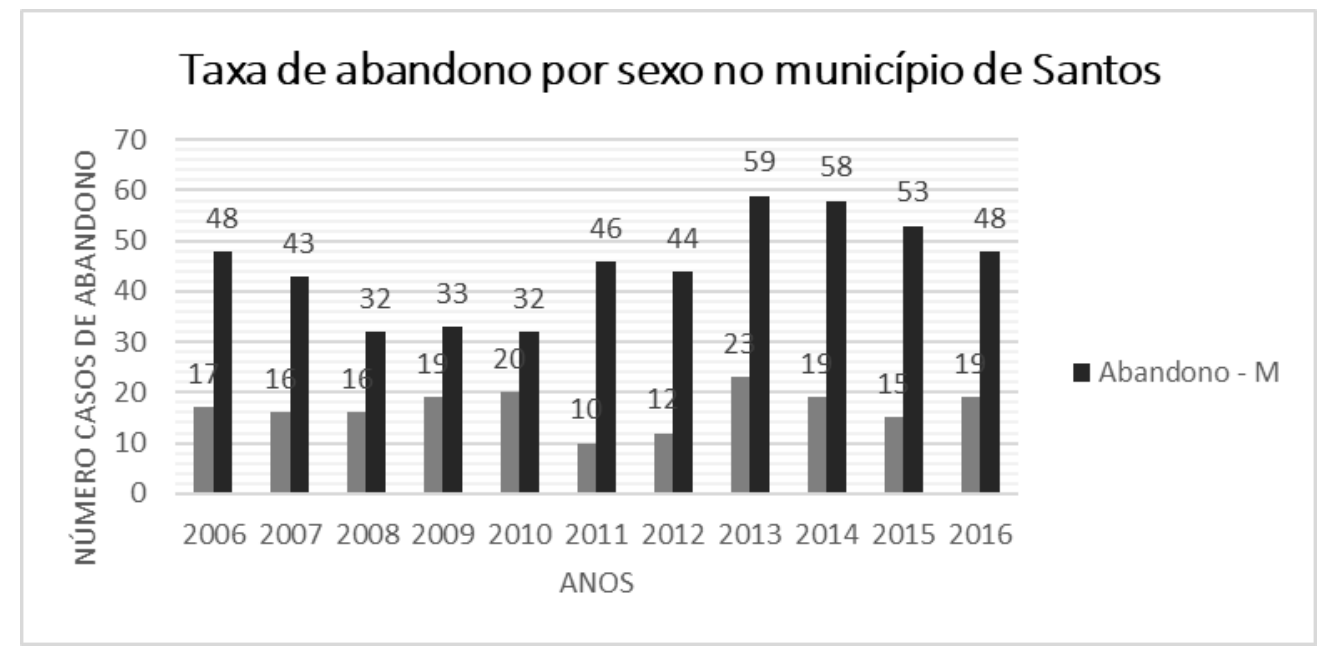

Fonte: Autores.

Uma análise do abandono de tratamento dividido por sexo no município de Santos (Figura 5) permite concluir que o abandono é muito maior na população masculina em comparação com a feminina, sendo maior que o dobro em alguns anos estudados, devemos levar em conta que a prevalência de Tuberculose na amostra selecionada é maior também na população masculina, impactando nos dados obtidos de abandono de tratamento. Nota-se uma elevação no número de casos de abandono 
na população masculina e uma certa estabilização da incidência entre as mulheres. Ao dividir a série histórica em dois momentos, observamos que nos seis primeiros anos houve uma redução do abandono entre os homens.

Levando em conta os bairros do município de Santos, foi possível realizar uma análise específica do número de casos para cada um dos bairros ao longo dos dez anos estudados na Figura 7. De todos os bairros da cidade os que apresentaram maior número de casos ao longo dos dez anos foram: Rádio Club (354), Vila Matias (257), Vila Nova (220), Boqueirão (217), Aparecida (202) e Macuco (202). A soma do número de casos desses 6 bairros selecionados representou aproximadamente $32 \%$ do total de casos (4533).

Figura 7 - Divisão dos casos de TB por bairros de Santos entre 2006 e 2016.

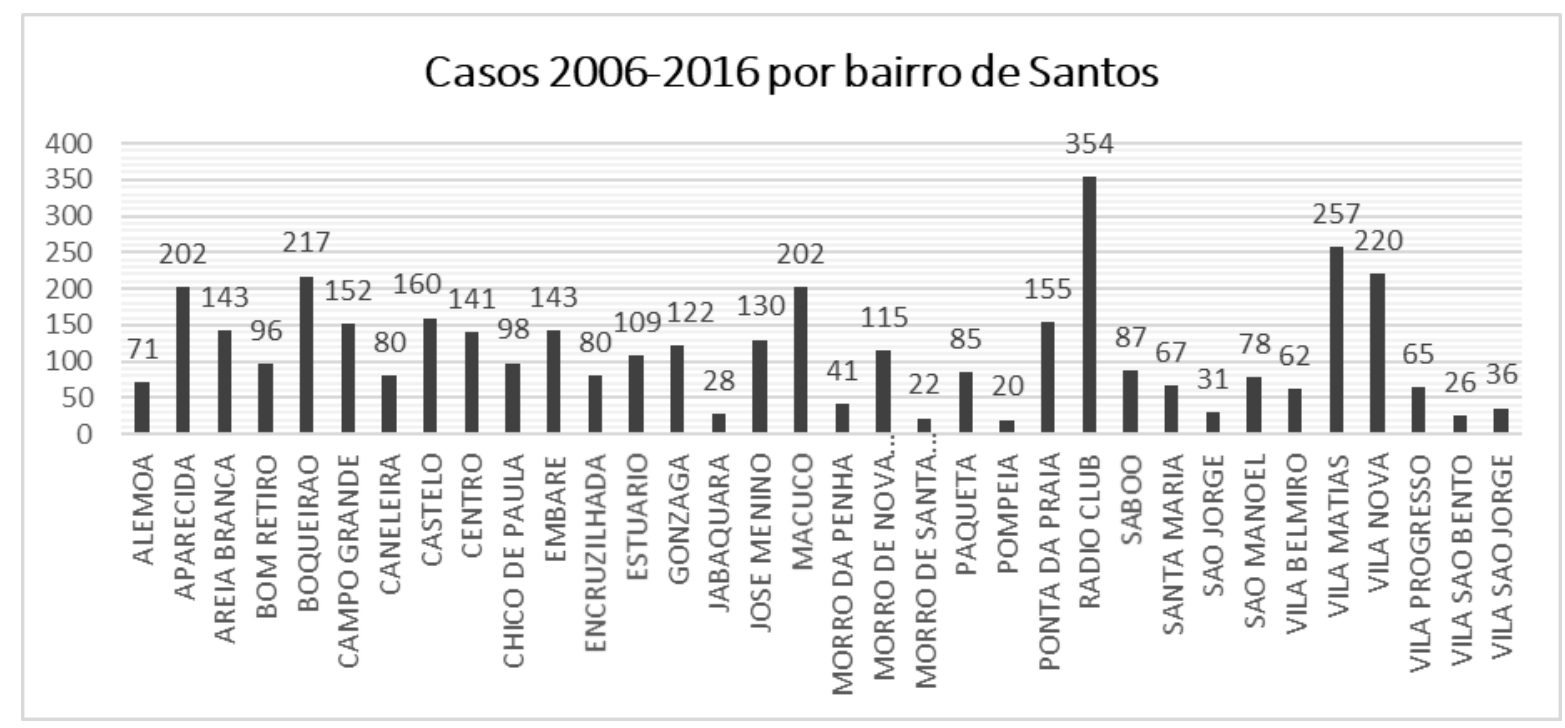

Fonte: Autores.

\section{Discussão}

Segundo a Secretaria de Vigilância em Saúde do Brasil, documento publicado em 2019 mostra que o coeficiente de incidência de Tuberculose no Brasil se encontra em 36,6 casos para cada 100.000 habitantes, representando uma tendencia de queda no comparativo com anos anteriores. Através de uma análise epidemiológica especifica para o município de Santos, observamos que entre os anos de 2006 e 2009 houve uma redução de 13\% nos casos de TB, seguido de um aumento de casos, o que resultou em uma elevação de 3\% nos casos para a década estudada. Esse resultado observado difere do que foi observado para toda a Baixada Santista onde foi observado um aumento de 18\% os casos para a população masculina e 19\% nos casos para a população feminina. Devemos levar em conta as diferenças populacionais, já que obtivemos uma amostra composta em 66\% de população masculina e 34\% de população feminina (Ministério da saúde, 2019).

Observamos que esse aumento no coeficiente de incidência observado no municio de Santos não condiz com o encontrado para todo território nacional. Durante o período estudado (2006 a 2016) o coeficiente de incidência Brasileiro apresentou uma redução de aproximadamente 4\%, já o coeficiente de incidência de Santos apresentou uma elevação de 3\%, contrapondo-se a tendencia de redução de casos nacional.

A revisão de literatura conduzida por CHIRINOS et al avaliou os fatores associados ao abandono de tratamento da Tuberculose, concluindo que, os principais fatores foram os sociodemográficos, uso de substâncias ilícitas e o nível de escolaridade (4). Levando em conta o desfecho obtido para cada um dos pacientes diagnosticados com Tuberculose, o grupo "ENCERRAMENTO" foi desenvolvido para estratificar as variáveis consideradas. Duas variáveis principais foram 
consideradas para análise, sendo elas "CURA" e "ABANDONO". Observamos que a taxa de cura representou aproximadamente $73 \%$ do total de desfechos obtidos, com 2 momentos epidemiológicos principais observados. Em um primeiro momento observamos uma redução nos índices de cura entre 2006 e 2009, seguida de um novo aumento nos índices entre 2009 e 2015, porém observamos uma redução nas taxas de cura para todo período analisado, com uma redução de $14 \%$ entre 2006-2016. Já a variável abandono 15\% dos desfechos (Chirinos et al, 2011).

Uma das grandes questões que contribuem para menores taxas de sucesso de tratamento está relacionada a taxa de abandono de tratamento segundo a biblioteca virtual em saúde. Levando em conta a taxa de abandono, fica evidente após avaliação dos resultados, que houve um aumento dessa taxa principalmente nos últimos anos avaliados pelo estudo com elevação ocorrendo principalmente entre 2008 e 2013. A distribuição da taxa de abandono, contudo, não foi equivalente entre as populações masculina e feminina, pois tanto o abandono quanto a incidência foram observados com maiores valores na população masculina. Foi observado certa estabilização dos casos de abandono entre as mulheres e com número de casos anuais menores ao comparar com os homens (Czeresnia, D et al 1985).

Devemos levar em conta que, para tanto, houve discrepância na amostra estudada no que diz respeito a quantidade populacional masculina e feminina. De todos os pacientes diagnosticados com Tuberculose, a população masculina representou $66 \%$ de toda amostra selecionada com TB, já a população feminina representou apenas $34 \%$. Sendo assim, tivemos uma amostra masculina quase duas vezes maior do que a feminina. Esses valores discrepantes na amostra incidem nos resultados obtidos para os cálculos comparativos entre sexos não apenas para taxa de Abandono, mas também nas análises de cura entre os sexos e índice de drogadição.

JUSTO et al, avaliaram a associação entre uso de drogas lícitas e ilícitas com Tuberculose, concluindo que houve uma associação estatisticamente significativa entre essas duas variáveis (5). Para a variável "DROGADIÇÃO", a análise comparativa entre a população com diagnostico de Tuberculose e uso de drogas mostrou que houve uma elevação acentuada do uso de drogas a partir de 2008. Importante salientar que um aperfeiçoamento do sistema de vigilância epidemiológica pode ter contribuído para esse aumento de casos por ano, não necessariamente uma elevação de incidência a partir de 2008 até 2016 (Justo et al, 2018).

Levando em conta a associação entre a Tuberculose e fatores sociais (Siqueira, 2014) (Fasca, et al 2008) (Sales, et al 2015) os índices comparativos de drogadição entre a população masculina e feminina, devemos levar considerar as diferenças populacionais de amostra apontadas nesse estudo. Nota-se que a população masculina apresentou índices mais elevado do que a feminina durante todo período avaliado e seu crescimento se deu de forma progressiva entre os anos de estudo, principalmente entre 2013 e 2014. Com relação a população feminina os primeiros casos observados aconteceram em 2010 e houve uma certa estabilização na incidência entre 2012 e 2015 com uma queda registrada em 2016, bem diferente do observado na população masculina, que apresentou elevação acentuada dos novos casos desde 2008. Importante levar em conta que uma maior capacidade de diagnóstico e acompanhamento dos pacientes pode ter contribuído para a elevação acentuada, principalmente na população masculina, das taxas de incidência, não representando uma elevação de casos novos, mas sim um aperfeiçoamento do sistema de vigilância epidemiológica.

Uma vez que o município de Santos possui uma grande heterogeneidade de sua população, tanto relacionado a sua composição quanto a distribuição no território, além de uma desigual distribuição de renda (Vicentin et al., 2002) (Sales \& Nunes, 2015), se faz necessário uma análise territorial dividida por bairros desse município. Avaliando cada bairro observamos uma grande disparidade de casos por ano para cada município, sendo que aquelas que apresentam maiores índices populacionais e de aglomeração populacional tiveram quantidade mais elevada de casos. Contudo, os bairros que obtiveram maiores taxas anuais de infecção por TB foram aqueles com maiores índices de pobreza, dentre eles: Radio Club (354 casos), Vila Matias (257 casos) e Vila Nova (220 casos), sendo que o bairro do Rádio Club foi um dos que apresentou maior 
incidência acumulada para o período estudado. Portanto estamos diante de duas grandes variáveis que contribuem para aumento no número de casos, sendo elas: Aglomeração populacional e pobreza.

\section{Conclusão}

Após análise dos resultados obtidos, podemos concluir que o município de Santos - SP apresentou uma variação no número de casos anuais ao longo do período estudado, com momentos de aumento e de redução no número de casos, mas com estabilização nos últimos anos avaliados.

Concluímos ainda que a taxa de abandono de tratamento foi sempre maior na população masculina comparada a feminina e que houve um aumento nos últimos anos da década avaliada, mas não representou um fator de risco relacionado a mortalidade por Tuberculose.

Por fim, ao avaliarmos a divisão dos números de casos por bairros no município, notamos que aquelas regiões com maior aglomeração populacional e com maiores índices de pobreza apresentaram porcentagens maiores de casos ao comparar com as demais localidades do município.

Para entender melhor a situação epidemiológica em que se encontra o município de Santos, acreditamos que seria necessária uma atualização dos dados da amostra obtida com dados mais recentes acerca da população acometida com Tuberculose associado a uma análise minuciosa dos fatores socioeconômicos encontrados em cada bairro do município de Santos.

\section{Agradecimentos}

Agradecemos ao nosso Orientador Marcos Montani Caseiro por todo carinho e suporte durante a confecção desta pesquisa, ao Centro Universitário Lusíada pelo apoio financeiro e ao Centro de Vigilância Epidemiológica do Estado de São Paulo (CVE-SP) pela base de dados fornecida.

\section{Referências}

Arakaki-Sanchez D., Brito R., C, Brazil, organizadores. Manual de recomendações para o controle da tuberculose no Brasil. Ministério da Saúde, Secretaria de Vigilância em Saúde, Departamento de Vigilância Epidemiológica; 2011. 284 p. (Série A. Normas e Manuais Técnicos).

Barcellos C, \& Santos S. M (1997). Colocando dados no mapa: a escolha da unidade espacial de agregação e integração de base de dados em saúde e ambiente através do geoprocessamento. Inf Epidemiol SUS;5(1):21-9.

Barreira, D. et al (2018) Os desafios para a eliminação da tuberculose no Brasil. Epidemiologia \& Serviços de Saúde, 27(1), 1-4.

Brasil. (2011), Ministério da Saúde. Secretaria de Vigilância em Saúde. Departamento de Vigilância Epidemiológica. Manual de recomendações para o controle da tuberculose no Brasil. Brasília: Ministério da Saúde;

Brasil. (2018), Ministério da Saúde. Secretaria de Vigilância em Saúde. Departamento de Vigilância Epidemiológica. Boletim Epidemiológico 37. Experiências de Programa de Controle da Tuberculose: Porque juntos iremos detectar, tratar e acabar com a tuberculose como problema de saúde pública no Brasil. Brasília: Ministério da Saúde; 2018.

Câmara G, Carvalho, M. S., Cruz O. G. \& Correa, V (2004). Análise espacial de áreas. Livro online.

Carvalho, F. A. F. T., et al (2011). A Tuberculose através dos séculos: Série Histórica para a mortalidade no município de Santos, São Paulo, Brasil, 18542010. Dissertação Mestrado em Saúde Coletiva. Santos: Universidade Católica de Santos;

Chirinos, N. E. C \& Meirelles, B. H. S (2011). Fatores associados ao abandono do tratamento da tuberculose: uma revisão integrativa. Texto contexto enferm;20(3):599-606.

Czeresnia, D et al, Considerações sobre a Tendência da Tuberculose no Brasil. Cad. Saúde Pública. 1985; 1 (3): $313-26$.

Fasca, S. F., et al (2008). Tuberculose e condições de vida: uma análise do Estado do Rio de Janeiro, Brasil, 2000 a 2002. Dissertação Doutorado em Ciências na Área de Saúde Pública. Rio de Janeiro: Escola Nacional de Saúde Pública, Fundação Oswaldo Cruz; 2008.

Hino, P et al. Padrões espaciais da tuberculose associados ao indicador adaptado de condição de vida no município de Ribeirão Preto (tese). Ribeirão Preto: Escola de Enfermagem de Ribeirão Preto da Universidade de São Paulo; 2008. 
Research, Society and Development, v. 11, n. 1, e24711124869, 2022

(CC BY 4.0) | ISSN 2525-3409 | DOI: http://dx.doi.org/10.33448/rsd-v11i1.24869

Justo, M. T, Lourenção, L. G., Sasaki N. S. G. M., dos S, Vendramini S. H. F., De Souza, N. G., \& Santos, M. de L. S. G. Associação entre tuberculose e consumo de drogas lícitas e ilícitas. EB. 23 de novembro de 2018;17(5):460

Lapa, T., Ximenes, R. A. A., Silva, N. N., Souza, W. V., Albuquerque, M. F. M., \& Cmpozana, G. Vigilância de Hanseníase em Olinda, Brasil, utilizando técnicas de análise espacial. Cad Saúde Pública. 17:11:53-62.

Mendes, A. M, \& Fensterseifer L. M (2004). Tuberculose: por que os pacientes abandonam o tratamento? Bol Pneumol Sanit [Internet]. http://http://scieloteste.iec.pa.gov.br/scielo.php?script=sci_arttext\&pid=S0103-460X2004000100005\&lng=en\&nrm=iso\&tlng=en

Organisation mondiale de la santé, organizador. Global tuberculosis report 2017. Geneva: World health organization.

Sales, C. M. M. Nunes, G. F. Rogério, W. Castro, T. Santos, B. R. \& Maciel, E. L. N. Tuberculose e a questão social: uma revisão sistemática de estudos brasileiros. In: Revista Brasileira de Pesquisa em Saúde. 17 (4): 156-175.

Siqueira, A. S. P. (2014) Determinantes socioeconômicos da produção da tuberculose: um estudo no município de Itaboraí, Região Metropolitana do Rio de Janeiro, no período de 2000 a 2011. Dissertação Doutorado em Ciências na Área de Saúde Pública. Rio de Janeiro: Fundação Oswaldo Cruz.

Souza, J. N., Bertolozzi, M. R et al (2007). A vulnerabilidade à tuberculose em trabalhadores de enfermagem em um hospital universitário. Ver Latino-am Enfermagem; 15(2):259-266

Vieira, A. G., et al. (2009) Características da tuberculose pulmonar em área hiper epidêmica - município de Santos (SP). Jornal Brasileiro de Pneumologia, 35

Vicentin, G.; Santo, A. H., \& Carvalho, M. S (2002). mortalidade por tuberculose e indicadores sociais no município do rio de janeiro. ciência \& saúde coletiva.

Ximenes, R. A. A., Martelli, C. M. T., Souza, W. V. et al, (1999) Vigilância de doenças endêmicas em áreas urbanas: a interface entre mapas de setores censitários e indicadores de morbidade. Cad Saúde Pública. 15:53-61. 